\title{
Diana Popović*
}

Université de Novi Sad, Serbie

\section{LA LANGUE ET LA QUÊTE D'IDENTITÉ DANS LA QUÉBÉCOITE DE RÉGINE ROBIN}

\section{Résumé}

Parmi les sujets les plus récurrents de La Québécoite on peut noter la langue (les langues), les souvenirs, l'espace, les racines, autrement dit tout ce qui fait la base de l'identité d'une personne immigrée. En tant qu'écrivaine migrante, Régine Robin a une sensibilité pour la condition de ceux qui ne se sentent pas à l'aise dans un nouveau pays, et elle nous peint, d'une manière tout à fait innovante, qui se veut une expérimentation sociale et littéraire, leurs angoisse et instabilité intérieures, qui se manifestent aussi au niveau de la langue. Dans le présent article nous nous proposons d'analyser le phénomène de la parole migrante, sa stratification (réduction aux fragments, aux listes etc.), son statut et ses rôles dans le texte robinien et de révéler ses rapports avec la quête identitaire du locuteur.

\section{Mots-clés}

Langue, identité, écriture migrante, roman québécois, Régine Robin.

* diana.popovic@ff.uns.ac.rs 


\section{Introduction}

Publié au Québec en 1983, le récit La Québécoite y a été bientôt marqué par un succès considérable et a été réédité dix ans plus tard. Ce roman au titre intriguant ${ }^{1}$, on l'a considéré très souvent comme « ethnique », vu le fait qu'il a été écrit en français par une écrivaine qui a eu une autre langue maternelle et qui n'était pas originaire du Québec. En tant qu'immigrée, Régine Robin y a traité du sujet qui lui était proche, du choc culturel et des difficultés pour une nouvelle arrivée de s'adapter à un nouveau pays. Ce roman est devenu en son temps une figure emblématique de l'écriture migrante, notion qui était à cette époque en train de naître.

En outre, ce texte représente une œuvre fascinante, non seulement du point de vue de son contenu très riche, mais aussi de sa structure fragmentaire et de son procédé littéraire tout à fait innovant. Le récit n'est pas linéaire et, d'après les mots de l'auteure elle-même, se voulait « une expérimentation à la fois littéraire et sociale » (Robin 1993 : 207) qu'on aurait du mal à saisir et à définir. Il s'agit d'une œuvre où, apparemment, on ne respecte aucun ordre, ni chronologique, ni spatial, ni logique. En effet, c'est un texte-patchwork créé de morceaux disposés pêle-mêle, où l'on peut trouver accumulées les bribes du présent et du passé, des flashbacks de l'histoire (personnelle), de l'Histoire (collective), des espaces réels (anciens et contemporains), des espaces imaginaires, toute une diversité de cultures, traditions, langues et littératures, une pluralité des voix narratives, des histoires probables, des destins incertains et ainsi de suite. Néanmoins, le lecteur ne doit pas négliger le fait qu'il y existe un fil qui tient ensemble tous ces fragments dits désordonnés, lesquels finalement construisent une certaine image (matérielle, culturelle) des espaces évoqués et donnent aussitôt une idée d'errance sans fin, puisque l'histoire de chaque chapitre s'achève au même endroit, pour recommencer l'histoire d'une manière différente. Toutes ces histoires probables et ce mouvement (extérieur) dans l'espace symbolisent une perpétuelle recherche et reflètent l'instabilité intérieure d'une immigrée. En effet, au cours de son déplacement constant (vagabondage autant spatial que temporel) elle « ramasse » une par une les pièces du puzzle identitaire. La problématisation de l'identité ici se présente comme un projet très complexe où la notion de la langue, ou plutôt de la pluralité des langues, ne peut pas être dissociée d'autres thèmes récurrents, tels que les origines personnelles (des héros, de l'écrivaine), les bagages de la mémoire ou bien l'espace (cf. Popović 2019) et le mouvement. Tout cela contribue à une idée selon laquelle la quête identitaire d'une personne immigrée se fait parallèlement dans plusieurs direc-

${ }_{1}$ Le nom dérivé du substantif Québécoise et de l'adjectif coi, coite qui en français de France signifie quelqu'un sous l'effet de la surprise, de la stupéfaction, de l'émotion, de la crainte, et en canadien-français cela veut dire calme, tranquille, muet. 
tions et que les langues qu'on parle participent sans cesse de ce déchirement intérieur qu'on éprouve. Ici la langue maternelle et les autres langues qu'on parle se codent et se décodent sans interruption. C'est un processus dynamique et sans fin qui est lié inévitablement à cette instabilité intérieure qu'on ne peut ni éviter ni éliminer. Le sentiment très fort de l'angoisse se trouve donc à la base du comportement de l'immigrant et dans ses manières d'utiliser la parole, que Régine Robin appelle la parole migrante.

\section{Stratification de la langue}

Dans La Québécoite, le sujet du trouble intérieur se manifeste au moins à deux niveaux : au niveau purement linguistique (le choix entre la langue maternelle et la langue - les langues - d'adoption, le choix du vocabulaire), et aussi au niveau du style de l'écrivaine. L'écriture non-linéaire, typique pour ce roman, démontre que la langue en tant que phénomène a perdu sa cohérence. Sa stratification (qui est en effet propre à la parole migrante) est nécessaire pour montrer la stratification identitaire du locuteur qui se trouve dans une société consommatrice (Frédéric 1991 : 494-496). Le discours robinien incorpore des éléments hétéroclites et se réduit donc aux fragments, aux bouts de texte qui se présentent comme un poème, puis aux citations, aux différentes listes, inventaires, catalogues, nomenclatures, aux phrases ou locutions elliptiques, aux associations libres des mots (selon leur forme et non selon leur sens) et ainsi de suite. Une telle absence de cohérence du discours, de la structure linguistique attendue ou de la linéarité traditionnelle du texte écrit, aboutit à un modèle d'écriture qui n'est stable que dans son instabilité, dont le but est d'illustrer le concept robinien donné au début du roman ( «Pas d'ordre. Ni chronologique, ni logique, ni logis. Rien qu'un désir d'écriture et cette prolifération d'existence » (Robin 1993 : 15). Par son essence, ce modèle d'écriture, basée sur une fragmentation constante, un désordre et non-linéarité du discours, reflète ce mouvement intérieur qui se nourrit du deuil et de l'angoisse, qui accompagnent sans cesse une femme exilée. Le fait est que la relation entre, d'un côté, la langue (soit parlée ou écrite) et de l'autre la quête identitaire du locuteur, est très étroite, voire imminente. En outre, elle est marquée, c'est-à-dire elle est bien visible dans la présentation même du texte écrit qui est, comme on l'a déjà mentionné, très variée dans le roman. Donc, le discours lui-même renforcé par sa représentation sur le papier dévoile l'état d'âme du personnage central du roman, qui est une immigrée et en même temps l'incarnation de l'auteure elle-même. Cela ouvre la possibilité d'une lecture autobiographique du roman et souligne le fait que la relation entre les idées exprimées dans le texte (dans la fiction) et la réalité est particulièrement proche. 


\section{La vie urbaine et la langue en tant que témoin de la recherche de l'identité}

L'identité de la protagoniste (qui n'est pas nommée et reste elle tout au long du roman) se définit par son origine juive et par son éducation française (ce qui est le cas de Régine Robin, dont le nom d'origine était Rivka Ajzersztejn). Elle s'installe à Montréal (comme l'a fait l'auteure) et a un désir très fort de trouver sa place dans ce nouveau monde. Le roman est divisé en trois parties et chacune d'elles est intitulée par les noms des quartiers montréalais : Snowdon, Outremont et Autour du marché Jean-Talon. Ce sont trois lieux où l'héroïne essaie de vivre et de s'adapter. Puisqu'elle n'arrive pas à le faire, elle prend l'avion pour rentrer à Paris.

La narratrice qui raconte l'histoire de son héroïne, qui mène, en effet, trois vies hypothétiques dans le roman, puisque c'est toujours elle qui vit trois fois sa vie dans les trois contextes différents, avec un partenaire imaginaire, chaque fois d'une origine différente, d'un statut différent et d'une éducation différente. Chaque variante de sa vie à elle aboutit à un échec personnel de s'adapter à l'entourage social et c'est la raison pour sa fuite. Elle quitte le nouveau monde qu'elle n'a pas réussi à comprendre, et finalement part pour son Europe natale.

La clé pour la compréhension du texte réside dans le fait que la protagoniste se sent exilée et solitaire, qu'elle éprouve un sentiment d'inquiétude, de douleur et d'angoisse issu de sa situation d'être une étrangère, d'être « une autre » par rapport aux gens parmi lesquels elle vit. Elle regrette qu'elle ne réussira jamais à devenir Québécoise :

Quelle angoisse [...] - Québécité - québécitude - je suis autre. Je n'appartiens pas à ce Nous si fréquemment utilisé ici - Nous autres - Vous autres. Faut se parler. On est bien chez nous - une autre Histoire - L'incontournable étrangeté. Mes aïeux ne sont pas venus du Poitou ou de la Saintonge ni même de Paris, il y a bien longtemps. Ils ne sont pas arrivés avec Louis Hébert ni avec le régiment de Carignan - Mes aïeux n'ont pas de racines paysannes. Je n'ai pas d'ancêtres coureurs de bois affrontant le danger de lointans portages. Je ne sais pas très bien marcher en raquettes, je ne connais pas la recette du ragoût de pattes ni de la cipaille. Je n'ai jamais été catholique. Je ne m'appelle ni Tremblay, ni Gagnon. Même ma langue respire l'air d'un autre pays. Nous nous comprenons dans le malentendu. (Robin 1993 : 53-54)

Dans ce passage la narratrice essaie d'analyser sa position dans la société. Elle constate que son identité est fortement marquée par la différence par rapport aux Québécois. Et cette suite de négations nous révèle que c'est vraiment difficile pour elle de se définir, autrement dit de dire ce qu'elle est en réalité. Bien évidemment elle souligne l'importance des aïeux, du nom de famille, des recettes, de la foi et ainsi de suite, mais dans son cas à elle, tout cela lui ne donne qu'un 
statut d'immigrée et en même temps un sentiment de non-appartenance à la société montréalaise. C'est la raison pour laquelle l'errance dans l'espace (dans ce chapitre en traversant Snowdon, et puis après d'autres quartiers de Montréal) fait surgir une autre idée, celle du déracinement. Et cela n'est qu'un reflet de son origine juive. Elle est marquée par l'appartenance à un peuple sans pays, peuple qui erre incessamment, sans s'enraciner dans un seul endroit (Popović 2019 : 95-99).

Elle mentionne souvent la destinée de son peuple, de même que celle de sa famille, de son nom. Elle constate sèchement, sans beaucoup d'élaboration, que certains faits lui sont pénibles : « Je n'ai pas d'aïeux. Tous morts à Auschwitz et avant anonymes, des petits, des obscurs, des sans-grades » (Robin 1993 : 188).

Cette angoisse se lie à son idée « d'apprivoiser » en quelque sorte la ville où elle vient de s'installer. On peut remarquer que son expérience personnelle et intime de la ville de Montréal est doublement orientée : d'un côté elle est visuelle et de l'autre sonore. Sans interruption elle écoute et regarde, et on peut même dire qu'elle engloutit à chaque instant toutes les brebis de la réalité urbaine, laquelle lui paraît toujours nouvelle, et elle commence à noter, donc à utiliser sa langue pour enregistrer tout ce qu'elle voit et entend, tout ce qu'elle remarque et identifie comme "différent », bien évidemment de son point de vue (puisqu'elle n'appartient pas aux autochtones) :

Noter toutes les différences. Ne rien laisser au hasard.

Surtout ne rien négliger.

Fixer le nom des partis politiques étranges venus d'ailleurs [...] (Robin 1993 : 182. C'est nous qui soulignons)

Puis elle explique à quoi sert cette obsession de noter tous les faits de son entourage, qui est d'ailleurs devenue son style d'écriture :

Oui, noter toutes les différences. Ne rien oublier, ni les marques de dentifrices, les chaînes de Barbecues, celles de Pizza, ni les marques de savon ou de lessive. Pénétrer l'étrangeté de ce quotidien. En exil dans ta propre langue. Le leurre de la langue. Ni la même, ni une autre.

L'AUTRE dans le MÊME,

L'inquiétante étrangeté d'ici. (Robin $1993: 183$ )

La narratrice très fidèlement entreprend cette opération de fixer tous les fragments de la vie quotidienne, mais elle tient aussi à expliquer le but de cette entreprise, qu'elle relie à « une nouvelle existence » :

Noter toutes les différences. Tout cela finirait bien par donner de la réalité, tout cela finirait bien par lui faire comprendre le Québec, et Montréal et le parler 
d'ici, tout cela finirait bien par comprendre la configuration d'une nouvelle existence. (Robin $1993: 191^{2}$ )

On peut deviner qu'il s'agit d'un processus assez lent mais nécessaire. De cette manière la narratrice veut s'aider à comprendre les mœurs, la tradition, en un mot la vie à la montréalaise. C'est pourquoi elle fait toutes ces listes, catalogues, nomenclatures tout au long du roman, afin d'énumérer, dans la mesure du possible, d'innombrables détails qui surgissent autour d'elle et qu'elle trouve d'une véritable importance pour sa propre adaptation à cette « nouvelle existence » qu'elle est obligée de vivre. Passionnée par cette entreprise, elle « ramasse » tout et partout. Elle veut attraper et même plus que cela, fixer d'une manière linguistique (et non autrement) tout ce qui lui passe devant les yeux et s'enfuit par la suite dans le sens spatial. Elle veut donc nommer les choses pour les éterniser. C'est la raison pour laquelle son texte abonde en substantifs et adjectifs, avec lesquels on désigne ou qualifie les choses. On peut remarquer que Robin n'insiste pas sur des verbes. Par contre, son texte se caractérise par des blancheurs (des marges), qui symbolisent les blancheurs, plutôt des trous dans la vie des gens immigrés (Joseph 2001 : 35). Cette déconstrucion de la langue (langue écrite) reflète une atmosphère d'errance où l'on trouve mélangés les lieux, les souvenirs, les temps (passé, présent). D'une manière presque automatique, la narratrice capture, confusément, tout ce qui est « offert » librement dans la vie, devant elle, dans cette ville tellement spécifique et dynamique. Et la ville, elle est d'après la narratrice non seulement très variée, mais aussi « schizophrène, clivée, déchirée », et également un «patchwork linguistique, bouille ethnique » (Robin 1993). Il s'agit d'une ville qui est pluridimensionnelle du point de vue social, une ville-caléidoscope dans tous les sens (Couillard 1983: 26). Mais même si la narratrice aime ce mélange des langues et des choses qu'on peut y trouver, elle sent qu'elle ne le comprend pas toujours, ce qui provoque chez elle un sentiment d'être exclue et bien solitaire et c'est pourquoi elle finit chacune de ses trois histoires par le même scénario, par son retour à Paris. Comme le constate Hélène Amrit, la narratrice, en tant qu'étrangère, ne possède pas les codes nécessaires pour la compréhension totale de son nouvel entourage (Amrit $2014: 270$ ) et finit par sombrer dans sa profonde solitude : « Je ne suis pas d'ici. On ne devient pas québécois. Prendre la parole, rendre la parole aux immigrants, à leur solitude » (Robin 1993 : 54). Et cette solitude est inévitablement liée à ce que Robin nomme « la parole migrante ». C'est la parole qui inquiète, qui pèse énormément. Voilà la comparaison qu'elle ajoute à ce propos tout en montrant la lourdeur du langage : « La parole immigrante comme un cri, comme la métaphore mauve de la mort, aphone d'avoir été criée » (Robin 1993 : 55).

${ }^{2}$ C'est nous qui soulignons. 
D'un autre côté, la narratrice analyse le code parlé de sa langue. Elle s'aperçoit qu'elle ne peut jamais cacher ses racines, son origine non-québécoise, puisque sa langue française n'est pas celle qu'on parle au Québec :

Il lui aurait fallu faire oublier sa trop visible 'francité', son accent où percerait sans qu'il y paraisse un je-ne-sais-quoi d'impérialisme culturel, ses années de Sorbonne, d'École normale supérieure, ses années de cursus honorum un peu trop parfait, faire oublier toutes ses parisianités. (Robin $1993: 105$ )

Même si elle parle français, un français très raffiné et pur, il s'agit d'un « autre français ». Donc, même sa propre langue la marque, la distingue et fait d'elle une femme différente, provoquant encore une fois ce sentiment pénible d'être exilée. Cette différence langagière est prise plusieurs fois dans le roman avec un ton ironique. Par exemple : «Les courses à faire. Pardon. Magasiner» (Robin 1993 : 187). Le fait qu'elle est francophone, donc, ne lui facilite point les choses, par contre c'est ce qui lui rappelle qu'elle est une personne autre, une étrangère, une exilée.

La narratrice parle à plusieurs reprises de l'exil, sujet qui lui est pénible, et son discours est toujours imprégné par ses émotions :

Illusions de l'enracinement... Restera l'exil ? L'éternel sentiment d'être ailleurs, déracinée. Montréal ou Paris, Budapest ou Jitomir ou New York. Les villes se cherchent et se répondent dans la nuit. Parfois elles se ressemblent. Quelle importance ! (Robin 1993 : 187)

D'un côté le lecteur comprend que la vie montréalaise est prise symboliquement. Mais d'un autre côté Montréal est ici associée à l'image d'un véritable collage de langues, ce qui établit un parallèle avec une pelote langagière personnelle propre aux gens immigrés qui, en général, parlent plusieurs langues dont chacune d'elles crée ses propres relations avec le passé ou le présent du locuteur en question. Les flashbacks nombreux dans le roman prouvent que la liaison entre le passé et la langue est très vive, constante, éternelle et participe à la construction des puzzles identitaires.

\section{Pluralité des langues en tant que signe de l'identité migrante}

Régine Robin insiste sur la pluralité des langues en tant qu'une des caractéristiques les plus naturelles d'un(e) exilé(e). En réalité elle parle le yiddish (qu'elle désigne dans ce roman comme la langue des morts, la langue de ses aïeux), puis le français de France, l'anglais, l'allemand, l'hébreu, le russe. Chaque langue a sa propre importance et valeur pour le locuteur, puisqu'elle dirige son comportement et plus généralement sa vie. Pour Robin, parler une langue c'est 
« se placer dans le temps ». Si l'on parle le yiddish, par exemple, cela veut dire parler du passé des siens. Cela se voit bien dans le passage suivant du roman :

Un vieux Juif revient dans son village natal après la guerre. Il est le seul survivant. Il rencontre une jeune fille, elle aussi survivante du massacre. Il lui parle en yiddish, elle traduit en russe. Quand elle lui demande si la traduction est bonne, il répond : "Que dire ? Les souffrances, elles, elles étaient en yiddish !» (Robin 1993 : 151)

La langue s'impose donc comme le miroir de la destinée d'un peuple. Robin mentionne ou utilise sa langue maternelle, le yiddish, et la met dans le contexte du passé de son peuple, de son Histoire collective. Tout le roman est écrit sous le signe de cette langue (yiddish), mais aussi de cette culture (Boutin-Panneton 2012 : 36). Robin révèle tout un contexte historique et même mythique de cette langue (Boutin-Panneton 2012: 40), et la colore des émotions, qui ne sont pas exclusivement les siennes, mais collectives et les images du sang et de la mort sont entrées pour toujours dans la mémoire collective des juifs. Leur langage est défini ici comme :

un langage à l'envers ${ }^{3}$, allant vers on ne sait quoi. Une image graphique qui est tout un paysage. Un langage sang, mort, blessure, un langage pogrom et peur. Un langage mémoire. (Robin 1993 : 140)

D'un autre côté, la pluralité des langues (de l'auteure elle-même et par conséquent de la narratrice du roman) se présente comme nécessaire pour la vie quotidienne montréalaise et pour ses habitants. À propos de l'anglais, le russe et la langue maternelle la narratrice dit : " Désormais, le temps de l'ailleurs de l'entre-trois langues, trois alphabets dans la même journée. » (Robin 1993 : 133), pour souligner qu'à Montréal il y a toute une gamme de langues. De même il y existe toute une variété de nations et leurs héritages divers s'entrelacent. Mais son cas à elle est toujours unique : elle se sent isolée et encore une fois se définit comme un être à part, comme un îlot dans la mer : «Exclue. Seule dans cette ville-collage, cette ville-livre, cette ville-Histoire. » (Robin 1993 : 144). Si cette pluralité des langues est vue comme un avantage pour une métropole, elle ne l'est pas pour une femme juive exilée. Cela lui donne de la peine. Cela lui rappelle son origine, son histoire personnelle et encore plus son Histoire collective qui sont toutes les trois très spécifiques, puisqu'elles sont liées inévitablement à la douleur d'un peuple errant.

On peut remarquer que tous les personnages du roman parlent au moins deux, ou très souvent plusieurs langages. La phrase suivante, prononcée par le professeur Mortre, démontre que le phénomène du plurilinguisme est omniprésent

3 À un autre endroit : «Un langage à l'envers, pas comme les autres, de droite à gauche. » (Robin 1993 : 139) 
dans le roman et que l'anglais s'y impose comme indispensable : « Si avec cinq langues je n'épuise pas le sujet, je givappe » (Robin 1993 : 42). La question qui se pose est si les langues, et surtout si elles sont au pluriel, suffisent pour s'exprimer. On dirait que non. Pour la narratrice, la langue en tant que phénomène, est le reflet de la réalité objective (ici d'une ville multiculturelle) et également de la réalité subjective (d'une expérience personnelle éprouvée par chaque individu qui vit dans cette ville même), et c'est la raison pour laquelle leur correspondance (des deux réalités) se fait d'une manière unique et propre pour chaque individu. Chaque langue y joue un rôle considérable, prend un rôle de fil conducteur entre le passé et le présent, entre l'expérience vécue et vivifiée dans un moment donné. Cela veut dire que l'identité migrante est un phénomène très complexe, vu le fait que l'homme, avec tout son passé vu et vécu, est comme une boîte à multiples fonds.

À cela s'ajoute la question du choix de la langue d'écriture chez les immigrants. Régine Robin trouve un moyen de s'exprimer non seulement en une langue, en français, mais aussi par un style d'écriture, qui devient un instrument par excellence pour « aborder une tension entre nous et les autres » et pour exprimer son identité « boiteuse, douloureuse, souffrante » (Verduyn 2000 : 75) qui loge dans son être le plus profond, « un être qui a en soi l'autre », ce que Robin appelle « dedans dehors » (Nepveu $1998: 35$ ).

\section{En guise de conclusion}

On peut constater que La Québécoite est un roman abondant en langues, où chacune d'elles a son statut et sa valeur, soit très subjective, personnelle ou générale, universelle, ce qui dépend de la position qu'elles occupent dans la vie personnelle d'un individu ou de la vie sociale en général. La pluralité des langues témoigne que même si une société est multiculturelle, telle que la société canadienne-française, elle connaît une certaine crise identitaire, puisque la division en deux langues officielles ne suffit plus. À part les anglophones et les francophones (ici représentés comme des habitants des deux parties de la ville où se passe les deux vies hypothétiques de la narratrice, Snowdon et Outremont) il y a des « autre-phones » (dans la troisième partie de la ville, située autour du marché Jean-Talon), ceux qui vivent partagés entre plusieurs idiomes, et qui veulent avoir leur place à eux dans la société. Une pluralité des langues signifie qu'il y a une pluralité des identités des gens qui s'en servent, et cela est la seule réalité pour eux, les immigrés, même s'ils arrivent de la France, pays francophone par excellence.

Régine Robin vivifie beaucoup de cultures et de mœurs ici, et critique la société montréalaise pour finalement ouvrir la question existentialiste qui suis-je ? La réponse y est complexe, et toujours liée à la question de la langue (des langues). Pour les immigrés, le phénomène de la parole migrante est une des questions 
fondamentales. Régine Robin la traite avec un dynamisme constant dont la base est bien ancrée dans le passé, mais qui balance toujours entre le passé et le présent. Pour une immigrée (l'héroïne et également l'auteure elle-même), il est issu d'une angoisse très forte et constante dont on ne peut jamais se sauver. Si l'on a l'impression de ne pouvoir s'enraciner dans le monde matériel (qui est palpable et spatial), le message du roman est qu'on est enraciné à jamais dans un autre monde, non-matériel, celui de sa propre langue, sa propre pluralité des langues.

La langue, soit maternelle ou une autre, fait partie indispensable de l'identité d'un individu, elle est son fond et dirige son destin, puisqu'on ne peut pas la négliger ni l'effacer. Pour la narratrice, « la langue-identité », pour utiliser le terme de Sandrine Joseph, c'est le yiddish, la langue de sa mère, la langue « d'avant la séparation et le désordre » et qui par la suite aide la narratrice à « suivre une route en constante mutation et menant elle ne sait où » (Joseph 2001 : 39). Au cours de son errance et de ses vies probables, le seul fait qui ne change jamais et qui est le pilier identitaire, c'est son origine et sa langue maternelle. Tout le reste est sans frontières et dans le domaine de la potentialité.

\section{BIBLIOGRAPHIE}

Amrit, Hélène. « La littérature migrante est-elle soluble dans l'autofiction ? ». Analyses, 2014, 9, 2, 248-275.

Boutin-Panneton, Sylvie-Anne. Analyse des figures féminines juives dans le roman québécois moderne et contemporain : L'avalée des avalés, La Québécoite et Hadassa. Montréal : Université de Montréal, 2012.

Couillard, Marie. «Une parole qui dérange : La Québécoite de Régine Robin ». Lettres québécoises, 1983, 31, 26-27.

Frédéric, Madeleine. «L'écriture mutante dans La Québécoite de Régine Robin ». Voix et Images, 1991, 16, 3, 493-502.

Joseph, Sandrina. « Désormais le temps de l'entre-deux. L'éclatement identitaire dans La Québécoite de Régine Robin ». Globe, 2001, 4, 1, 29-51.

Nepveu, Pierre. Intérieurs du nouveau monde : essais sur les littératures du Québec et des Amériques. Montréal : Éditions du Boréal, 1998.

Popović, Diana. «Prostor i konstrukcija identiteta u romanu Lutalica (La Québécoite) Régine Robin ». Književna smotra, 2019, 192, 2, 95-101.

Robin, Régine. La Québécoite. Montréal : XYZ éditeur, 1993.

Verduyn, Christl. «L'éth(n)ique, La Québécoite et l'écriture migrante québécoise ». Tessera, 2000, 29, 73-81. 